\title{
The Salpeter plasma correction for solar fusion reactions
}

\author{
J. N. Bahcall ${ }^{1}$, L. S. Brown ${ }^{2}$, A. Gruzinov ${ }^{3}$, and R. F. Sawyer ${ }^{4}$ \\ 1 Institute for Advanced Study, Princeton, NJ 08540, USA \\ e-mail: jnb@ias.edu \\ 2 Department of Physics, University of Washington, Seattle, Washington 98195, USA \\ e-mail: brown@.phys . washington.edu \\ 3 Institute for Advanced Study, Princeton, NJ 08540, USA \\ e-mail: andrei@ias.edu \\ 4 Department of Physics, University of California at Santa, Barbara, Santa Barbara, CA 93106, USA \\ e-mail: sawyer@sarek.physics.ucsb.edu
}

Received 1 August 2001 / Accepted 3 December 2001

\begin{abstract}
We describe five different ways of obtaining the Salpeter formula for the plasma corrections to fusion rates; this formula is valid at the center of the sun with insignificant errors ( $\sim$ percent). Several recent papers have obtained a variety of answers in conflict with this result. We analyse the arguments of these papers, identifying sources of the discrepancies in some cases, and pointing out internal inconsistencies in the arguments given in other cases.
\end{abstract}

Key words. nuclear reactions - plasmas - sun: interior

\section{Introduction}

The plasma in the core of the sun is sufficiently dense that non-ideal gas corrections to nuclear reaction rates are significant. The plasma coupling parameter is given by $g=\left(e^{2} / D\right)(1 / k T)$, in which $T$ is the temperature of the plasma and $D$ is the Debye length defined by a sum over all the plasma ions and electrons,

$D^{-2}=\sum_{a} \frac{4 \pi e_{a}^{2} n_{a}}{k T}$

where $e_{a}$ and $n_{a}$ are the charge and density of the species labeled by $a$. The parameter $g$ is the ratio of the Coulomb potential energy for two particles a Debye length apart to the kinetic energy in the plasma. Near the center of the sun, $g \simeq 0.04$.

Recently, there have been a number of papers (Carraro et al. 1988; Shaviv \& Shaviv 1996, 1997, 2000; Savchenko 1999, 2001; Tsytovich 2000; Opher \& Opher 2000a, 2000b; Lavagno \& Quarati 2000; Weiss et al. 2001) suggesting that the standard screening corrections, originally derived by Salpeter (1954), need to be replaced by some other plasma physics correction, and that moreover the changes

Send offprint requests to: R. F. Sawyer, e-mail: sawyer@vulcan.physics.ucsb.edu could lead to substantial improvements in the standard solar model and the predicted solar neutrino fluxes.

The motivation for many of these papers is to "solve the solar neutrino problem" without invoking new weak interaction physics, such as neutrino oscillations. However, the results of solar neutrino experiments cannot be accounted for in this manner even if one goes to the extreme limit of treating the nuclear reaction rates as free parameters (see, e.g., Bahcall et al. 1998; Hata et al. 1994; Heeger \& Robertson 1996). Some distortion of the energy spectrum of electron type neutrinos is required.

The purpose of this paper is to highlight the compelling evidence for the Salpeter screening formula under the conditions that are relevant at the center of the sun, i.e., in the limit of weak screening. A major goal is to show that a necessary (but not sufficient) condition for the validity of a screening calculation is that the calculation must yield the Salpeter result in the limit when $g$ is very small. We also point out errors in some of the recent treatments of screening. The raison d'etre for our paper is the requests that we have had from colleagues for a written response to the numerous papers claiming large new effects (all different) in the calculation of solar fusion rates.

We summarize in Sect. 2 the results of five different derivations that all yield the Salpeter formula for 
screening. In Sect. 3, we describe briefly the flaws that lead to five different, non-Salpeter screening formulae. We summarize our principal conclusions in Sect. 4 .

\section{Five derivations that yield the Salpeter formula}

\subsection{Salpeter electrostatic derivation}

As shown by Salpeter (1954), fusion rates are enhanced by electrostatic screening. Here is the physical plausibility argument used by Salpeter.

If one of the fusing ions has charge $Z_{1} e$, it creates an electrostatic potential $\phi_{1}=\left(Z_{1} e / r\right) \exp (-r / D)$, where $r$ is the distance from the ion, and $D$ is the Debye radius. For $r \ll D, \phi_{1}=Z_{1} e / r-Z_{1} e / D$ is the Coulomb potential minus a constant potential drop. This potential drop increases the concentration of ions $Z_{2}$ in the neighborhood of $Z_{1}$ by the Boltzmann factor $\left[\propto \exp \left(-Z_{2} e \phi_{1} / k T\right)\right.$, where $T$ is the plasma temperature]

$f_{0}=\exp \left(\frac{Z_{1} Z_{2} e^{2}}{k T D}\right)=\exp \left(Z_{1} Z_{2} g\right)$,

Eq. (2) is the Salpeter formula. According to Salpeter, the quantity $f_{0}$ is the ratio of the true reaction rate to the reaction rate calculated using the ideal gas formula.

Salpeter's derivation makes physically clear that electrostatic screening causes an enhancement in the density of fusing partners by lowering the potential in the vicinity of a fusing ion. We shall come back to this physical argument in Sect. 3.6.

\subsection{WKB derivation}

The correction due to screening can be derived by calculating the barrier penetration factor in the presence of a plasma. Bahcall et al. (1998) evaluated the barrier penetration for a Debye-Huckel plasma. They showed that the usual Gamow penetration factor $e^{-2 \pi \eta}$, where $\eta=Z_{1} Z_{2} e^{2} / \hbar v_{\text {rel }}$ with $v_{\text {rel }}$ the relative velocity of the reacting ions, is replaced by

$\Gamma(E)=e^{-2 \pi \eta} e^{x \pi \eta}$.

Here $x=x(E)=R_{\mathrm{c}} / D$, where $D$ is again the DebyeHuckel radius, $R_{\mathrm{c}}$ is the classical turning-point radius defined by

$V_{\mathrm{sc}}\left(R_{\mathrm{c}}\right)=\frac{Z_{1} Z_{2} e^{2}}{R_{\mathrm{c}}} e^{-R_{\mathrm{c}} / D}=E$,

and $E$ is the relative energy of the reacting ions. Averaging $\Gamma(E)$ over a Maxwell-Boltzmann distribution, the effect of $e^{x \pi \eta}$ is just to multiply the total reaction rate by the Salpeter correction $f_{0}$.

The WKB has an advantage over the original Salpeter derivation that it shows explicitly how the Coulomb wave functions naturally lead to the Salpeter formula. This derivation is more rigorous than the Salpeter formulation, but is perhaps less transparent physically. The WKB derivation shows that the Salpeter formula is valid in the moderate density limit in which Debye-Huckel screening is a good approximation to the charge density distribution.

\subsection{Density matrix derivation}

Gruzinov \& Bahcall (1998) calculated the electron density in the vicinity of fusing nuclei using the partial differential equation for the density matrix that is derived in quantum statistical mechanics. This is the first calculation to describe properly the electron density close to the fusing nuclei. Given the electron density, Gruzinov \& Bahcall then evaluated screening corrections in a mean field approximation by solving numerically the PoissonBoltzmann equation for a mixture of electrons and ions. The electron density distribution obtained from the density matrix calculation was included self-consistently and iteratively in the mean field equation.

The mean-field calculation yields exactly the Salpeter result $f_{0}$ in the limit of low density. Higher order screening corrections were evaluated and found to be of order $1 \%$ for all of the important solar fusion reactions.

The density matrix formulation has the advantage over the WKB method and the Salpeter original derivation in that it is independent of assumptions about the original basis functions, while at the same time allowing the first proper calculation of the electron density distribution close to the nuclei. In addition, the density matrix formulation provides a formal procedure for evaluating corrections to the Salpeter formula.

\subsection{Free-energy calculation}

Dewitt et al. (1973) gave a rigorous derivation of the fusion rate corrections in the weak screening limit based on the free energy of fusing ions. Stimulated by one of the incorrect derivations of screening corrections, Bruggen \& Gough (1997) explained why the free energy is useful in this context.

For a given relative position of the two ions, one considers the electrostatic contribution to free energy from the rest of the plasma. In fact, it is sufficient to calculate the free energy correction for a single charge $Z, \delta F(Z)$. Then the rate enhancement factor is

$f_{0}=\exp \left(\frac{-\delta F\left(Z_{1}+Z_{2}\right)+\delta F\left(Z_{1}\right)+\delta F\left(Z_{2}\right)}{T}\right)$.

In the limit of small plasma density - the weak screening limit - this free energy correction can be calculated exactly. The result is the Salpeter formula.

Equation (5) has the physically obvious characteristics that the enhancement is symmetric in $Z_{1}$ and $Z_{2}$ and goes to zero in the limit of $Z_{1}$ or $Z_{2}$ going to zero. We shall come back to these physically obvious characteristics in Sect. 3.6.

The free-energy calculation is only as accurate as the expression for the free-energy. The physics that is contained in the approximation that has been used by 
Dewitt et al. and by Bruggen \& Gough is not more accurate than the physics contained in, for example, the density matrix derivation described in Sect. 2.3. An advantage of the free energy derivation is that it represents a valid derivation involving an independent technique.

\subsection{Quantum field theory derivation}

Brown \& Sawyer (1997) have developed a rigorous, general formulation for calculating the rate of fusion reactions in plasmas. The Brown-Sawyer formalism can be used to develop an unambiguous perturbation expansion in the plasma coupling parameter $g=e^{2} / D k T$. The general formula derived in Brown \& Sawyer (1997) reduces to the Salpeter correction to first order in $g$. Since $g=0.04$ at the center of the sun, this correction should suffice for solar model calculations.

The Salpeter effect is formally of order $e^{3}$. The first correction terms to it, which are formally of order $e^{4}$, are also examined in Brown \& Sawyer (1997). The largest correction at this order comes from the fact that the electrons are slightly degenerate, so that the first-order effects of Fermi statistics must be retained. This small effect is explicitly computed in Brown \& Sawyer (1997). The remaining terms of the formal $e^{4}$ order are of relative order $\ell / D$ to the basic Salpeter correction, where $\ell$ is either a ionic thermal wavelength $\lambda$ or the Coulomb classical turning point $R_{\mathrm{c}}$ of the fusing ionic motion. An upper bound shows that these are negligible contributions. These higher-order calculations provide evidence, which goes beyond the qualitative statement that the plasma is "weakly coupled", that the Brown-Sawyer perturbation expansion is applicable in the solar domain.

This method contains all of the physics described in the previous four derivations and provides a formal validation of the other techniques for obtaining the Salpeter formula.

\section{Papers claiming that Salpeter formula does not work}

\subsection{Dynamic screening}

"Dynamic screening" (Carraro et al. 1988) is a generic name for attempts to rectify the following perceived defect in the Salpeter argument: approximately one-half of the squared Debye wave number $D^{-2}$ comes from screening by electrons in the plasma and one-half from ions. As the two nuclei that are to fuse approach each other, the electron speeds are fast enough for the electronic cloud to adjust to the positions of the nuclei. But the by-standing ions in the plasma may be thought to have a problem making the adjustment, since their speeds are of order of the speeds of the ions that are to fuse. Thus it could appear that the effects of the ionic screening could be less than those in the Salpeter result, with a consequent reduction in fusion rates.

However, Gruzinov (1998, 1999) gave a general argument showing that in an equilibrium plasma there is no such reduction. For the Gibbs distribution (probability of a state being occupied proportional to $\exp (-\operatorname{energy} / k T))$, momentum and configuration probabilities are independent, and velocities of fusing particles have no effect on screening.

Moreover, in the most straightforward model for "dynamical" screening, the model advocated by Carraro et al. (1988), one can see how the "dynamical" part of the correction terms get canceled. The paper calculates a modified potential function for the fusing ions by following the motion of test bodies with positive charge approaching one another in a plasma characterized by the standard dynamic plasma dielectric constant. This modified potential produces corrections in fusion rates that are perturbatively of order $e^{3}$ times the uncorrected rates, as are the Salpeter effects when expanded. However, in Appendix D of Brown \& Sawyer (1997) it is shown that resulting modifications of the Salpeter result are exactly canceled (to order $e^{3}$ ) when processes are included in which the plasma has been excited or de-excited in a Coulomb interaction with one of the incoming ions. This is the reason that a calculation based only on a modified potential for elastic scattering fails.

To summarize, "dynamical screening" results, both in their simple realization in Carraro et al. (1988), and in any calculation that implements the qualitative argument given above, are refuted both by the general argument regarding the factorizability of the distribution function and by explicit calculation.

\subsection{Dynamic effect of Shaviv \& Shiviv}

Shaviv \& Shaviv $(1997,2000)$ state that a significant dynamic effect on screening does exist. We have not been able to extract from their work a single definitive analytic formula to compare with the Salpeter result, or with the various results of the rest of the heterodox "screening" literature. We can comment on some of their qualitative statements, with the caveat that we are not always sure how they have been implemented. The statements that we discuss below come from a recent summary "Why the Salpeter screening formula cannot be applied in the Sun", Shaviv (2000).

"Kinetic equations must be used to solve the screening problem." "Some reactions are enhanced and others suppressed depending on... Gamow energy..." In reality, kinetic effects are negligibly small in the Sun (because the classical turning radius at Gamow energies is much smaller than the mean particle separation or Debye radius). One should use equilibrium statistical mechanics to find initial conditions for binary collisions that lead to fusion. "All treatments of the screening... are based on the mean field approximation." This is not correct. The quantum field treatment, described in Sect. 2.5, makes no use of a "mean field" approximation superimposed on the equations, only a straightforward perturbation expansion of these equations. 


\subsection{Unconventional interpretation of the Gibbs distribution}

Opher \& Opher (2000b) propose, in order to support their version of dynamic screening, a different interpretation of the Gibbs probability distribution than is given in any standard treatments of statistical mechanics. In a discussion using classical (i.e., non-quantum mechanical) concepts, they argue that the Gibbs distribution may not be decomposable into uncoupled position and velocity factors; rather that the momentum variables must be regarded as position dependent. This assumption appears to confuse the concept of a trajectory with that of a probability distribution.

This assumption contradicts one of the foundations of statistical mechanics, as can be seen by consulting any of the standard expositions of the subject. For example, Landau \& Lifshitz (1996) stress: “...the probabilities for momenta and coordinates are independent, in the sense that any particular values of the momenta do not influence the probabilities of the various values of the coordinates, and vice versa".

The idea that coordinates and their conjugate momenta are independent statistical variables is familiar from elementary quantum mechanics where one calculates the phase space for a free particle as proportional to $\mathrm{d}^{3} x \mathrm{~d}^{3} p$, the product of the differential volume in space and the differential volume in momentum.

\subsection{Cloud-cloud interaction}

Shaviv \& Shaviv (1996) claimed that the screening energy $Z_{1} Z_{2} e^{2} / D$ which appears in the exponent of Salpeter's formula should be multiplied by a factor of $3 / 2$. They argued that a proper inclusion of the electrostatic interactions involving the screening clouds surrounding both fusing ions should lead to a modification of the Salpeter formula. Bruggen \& Gough (1997) have analyzed this claim in detail, and conclude that the result comes from the use of an incorrect interpretation of the thermodynamics, leading to an incorrect potential in the Schrödinger equation used for the system ${ }^{1}$.

\subsection{Unconventional statistics}

There are claims in the literature (see e.g., Savchenko 1999, 2001; Lavagno \& Quarati 2000) that the usual Salpeter expression does not apply because standard statistical mechanics (Gibbs distribution) is not valid; different statistical distributions are proposed. There are at least three reasons why these (and other authors) suppose that the Gibbs distribution is not valid in the sun. These three reasons are: 1) perhaps there is not enough time for

\footnotetext{
1 Although Bruggen \& Gough (1997) reach the right conclusion on this one point, their statement that the "dynamic screening" of Carraro et al. (1988) is needed when the reacting ions are fast in comparison with the screening particles is wrong, as we have emphasized in Sect. 3.1.
}

statistical equilibrium to be established; 2) perhaps there are interactions which distort the phase space distribution; and 3) perhaps the Gibbs distribution is not the correct equilibrium distribution. We discuss these three possibilities in the following subsections.

\subsubsection{There is not enough time}

Some of the suggested distributions seem to be based upon the assumption that the core of the Sun is not in thermodynamic equilibrium, and that there exist deviations from the Gibbs distribution. Both analytic calculations and Monte Carlo simulations show that the energy distribution of ions in a plasma rapidly approaches a Gibbs distribution on the time scale for the exchange of a major fraction of the typical particle energy among the interacting ions (see, e.g., MacDonald et al. 1957).

There is a slight departure from statistical equilibrium in the energy distribution of ions in the solar core, but the magnitude of the effect is too small to be of significance for any measurable quantity. The burning of nuclei in the sun is a non-equilibrium process, which causes a departure from the ideal Gibbs distribution. The magnitude of the deviation, $\delta$, is of order the Coulomb collision time $\tau_{\text {Coulomb }}$ over the nuclear burning time $\tau_{\text {nuclear }}$, Bahcall (1989). For the solar core,

$$
\begin{aligned}
\delta= & \frac{\tau_{\text {Coulomb }}}{\tau_{\text {nuclear }}}=10^{-28}\left[\left(\frac{\tau_{\text {nuclear }}}{10^{10} \mathrm{yr}}\right)\right. \\
& \left.\times\left(\frac{20 \mathrm{keV}}{E}\right)^{3 / 2}\left(\frac{\rho}{150 \mathrm{~g} \mathrm{~cm}^{-3}}\right)\right]^{-1} .
\end{aligned}
$$

The characteristic times for the most important solar fusion rates range from $10^{2} \mathrm{yr}$ to $10^{10} \mathrm{yr}$. Thus the number in the square brackets above is not significant in comparison to the overall factor of $10^{-28}$. For purposes of calculating solar fusion rates, the solar interior is in almost perfect thermodynamic equilibrium.

\subsubsection{Phase space distortion}

The rate $R$ for a binary nuclear reaction can be written symbolically as

$$
R \propto \iint \mathrm{d}^{3} p_{1} \mathrm{~d}^{3} p_{2} \exp (-E / k T)|\langle f|H| i\rangle|^{2} .
$$

The term $\mathrm{d}^{3} p_{1} \mathrm{~d}^{3} p_{2}$ in Eq. (7) represents the free-particle density of states calculated when the particles are very far separated. The Gibbs distribution is represented by the exponential, and the interactions are described by the matrix element of the Hamiltonian between initial and final states, $|\langle f|H| i\rangle|^{2}$.

The basic error made by some authors (see e.g. Savchenko 1999) is to confuse the role of the density of states, which can be calculated when the particles are at very large separations $\left(\mathrm{d}^{3} p_{1} \mathrm{~d}^{3} p_{2}\right)$, with the role of the interactions $\left(|\langle f|H| i\rangle|^{2}\right)$, which occur when the particles are very close together. 


\subsubsection{The Gibbs distribution is not the correct equilibrium distribution}

Many areas of modern physics, including large branches of condensed matter physics, as well as many classical subjects are successfully described by conventional statistical mechanics. There is no convincing evidence for any phenomena that lie outside the domain of standard statistical theory, which is described in the classical works of, e.g., Tolman (1938), Feynman (1972), and Landau \& Lifshitz (1996).

\subsection{Tsytovich suppression}

Tsytovich (2000) suggests that plasma effects lead to suppression rather than enhancement of fusion rates. Even though one point of the present note is to emphasize the need for careful analytic formulation in preference to plausible "physical" arguments, it is hard to differ with the qualitative result, in the original Salpeter discussion, summarized in Sect. 2.1, that screening enhances the reaction rate by lowering the potential in the vicinity of the fusing ions. Unfortunately, we do not understand the details of the elaborate treatment of Tsytovich well enough to pinpoint the difficulty.

However, since the treatment of Tsytovich is intended to be very general, one may consider a limiting case in which very large impurities of charges $\pm Q$ are introduced into a plasma undergoing $p-p$ fusion. The impurity charges are hypothesized to be so large that they dominate over electrons and protons in the electrostatic interactions. In these circumstances protons will preferentially clump around negative charges $-Q$. Locally, the proton density will increase and fusion will proceed faster. In this case, just as in the general case discussed by Salpeter, electrostatic screening enhances rather than suppresses fusion.

The result of Tsytovich (2000) also does not pass an even more basic check. In the weak screening limit, the Salpeter formula can be written as

$f_{0}$ (Salpeter $)=1+g Z_{1} Z_{2}$

where $g$ does not depend on the charges of the reacting particles $Z_{1}, Z_{2}$. In the limit when one of the reacting particles has a vanishingly small charge, the Salpeter screening effect goes to zero, that is the screening enhancement $f_{0}=1$. The Tsytovich formula has a different structure,

$f($ Tsytovich $)=1-g_{1} Z_{1}^{2}-g_{2} Z_{2}^{2}$,

which is a compact and revealing way to write Eq. (11) of Tsytovich (2000). Thus, $f$ (Tsytovich) $\neq 1$ if one of the particles is neutral, which is obviously incorrect.

\section{Summary and discussion}

All of the five ways of obtaining the Salpeter formula that are described in Sect. 2 are valid in the limit of a weakly coupled plasma. The different arguments are complimentary in this limit. The advantage of multiple derivations that use different physical concepts is that various authors or readers come to the subject of plasma corrections to fusion reactions with very different backgrounds and perspectives. Therefore, different individuals will find different arguments simpler or clearer.

In recent years, a number of authors have given alternative expressions, each different from all the others, for the weak field screening limit. We have described briefly in Sect. 3 some of the mistakes that seem to have been made in the papers that propose replacements of the Salpeter formulae.

As particularly emphasized in Sect. 2.5, the Salpeter result is the first term in a power series expansion in the plasma coupling parameter $g$. Near the center of the sun, $g \simeq 0.04$. Hence workers who claim that the Salpeter result is not valid for the sun must show that (a) their result approaches that of Salpeter when $g$ becomes arbitrarily small and (b) explain why their result significantly modifies that of Salpeter even though the perturbative parameter $g$ is much less than one.

\section{References}

Bahcall, J. N. 1989, Neutrino Astrophysics (Cambridge: Cambridge University Press)

Bahcall, J. N., Chen, X., \& Kamionkowski, M. 1998, Phys. Rev. C (Brief Reports), 57, 2756

Bahcall, J. N., Krastev, P. I., \& Smirnov, A. Yu. 1998, Phys. Rev. D, 58, 096016

Brown, L. S., \& Sawyer, R. F. 1997, Rev. Mod. Phys., 69, 411 Bruggen, M., \& Gough, D. O. 1997, ApJ, 488, 867

Carraro, C., Schäfer, A., \& Koonin, S. E. 1988, ApJ, 331, 565

Dewitt, H. E., Graboske, H. C., \& Cooper, M. S. 1973, ApJ, 181,439

Feynman, R. P. 1972, Statistical Mechanics: A Set of Lectures (New York: Addison-Wesley)

Gruzinov, A. V. 1998, ApJ, 496, 503

Gruzinov, A. V. 1999, preprint [astro-ph/9909272)]

Gruzinov, A. V., \& Bahcall, J. N. 1998, ApJ, 504, 996

Hata, N., Bludman, S., \& Langacker, P. 1994, Phys. Rev. D, 49, 3622

Heeger, K. M., \& Robertson, R. G. H. 1996, Phys. Rev. Lett., 77,3720

Landau, L. D., \& Lifshitz, E. M. 1996, Statistical Physics (Oxford: Butterworth-Heiman)

Lavagno, A., \& Quarati, P. 2000, Nucl. Phys. B (Proc. Suppl.), 87,209

MacDonald, W. M., Rosenbluth, M. N., \& Chuck, W. 1957, Phys. Rev., 107, 350

Opher, M., \& Opher, R. 2000a, preprint [astro-ph/0006326]

Opher, M., \& Opher, R. 2000b, ApJ, 535, 473

Salpeter, E. E. 1954, Aust. J. Phys., 7, 373

Savchenko, V. I. 1999, preprint [astro-ph/9904289]

Savchenko, V. I. 2001, Phys. Plasmas, 8, 82

Shaviv, G., \& Shaviv, N. J. 2000, ApJ, 529, 1054

Shaviv, N. J., \& Shaviv, G. 1997, Ap\&SS Lib., 214, 43

Shaviv, N. J., \& Shaviv, G. 1996, ApJ, 468, 433

Shaviv, G. 2000, preprint [astro-ph/0010152]

Tolman, R. C. 1938, The Principles of Statistical Mechanics (Cambridge: Cambridge University Press)

Tsytovich, V. N. 2000, A\&A, 356, L57

Weiss, A., Fleiskamp, M., \& Tsytovich, N. 2001, A\&A, 371, 1123 